\title{
An On-line Monitoring Technique for Electrode Degradation in Bio-Fluidic Microsystems
}

\author{
Q. Al-Gayem ${ }^{1}$, H. Liu ${ }^{2}$, A. Richardson ${ }^{1}$, N. Burd ${ }^{1} \&$ M. Kumar ${ }^{1}$ \\ ${ }^{1}$ Centre for Microsystems Engineering, Lancaster University, Lancaster, UK; ${ }^{2}$ Moor Instruments \\ Ltd, Axminster, UK. \\ \{q.al-gayem,a.richardson,n.burd,m.kumar\}@lancaster.ac.uk
}

\begin{abstract}
This paper presents a solution for detecting degradation in electrodes that interface to fluidic or biological systems that forms the basis of numerous actuation and sensing mechanisms in the bio-fluidics field. In this solution, a mid-frequency oscillation test strategy is proposed and evaluated experimentally on an array of electrodes. This technique is based on the sensitivity of the bio-fluidic interface capacitance to degradation, contamination and fouling.
\end{abstract}

\section{Introduction}

Microfluidics technology that has the potential to stimulate the integration of silicon based sensors, electronics, and fluidic functions for biochemical analysis within portable miniaturized bio-fluidic instruments. The market sectors of specific interest include medical diagnostics, industrial control, environmental monitoring and pharmacology. Examples are DNA extraction from blood, detection of pathogens, e.g. genetically modified organisms (GMO's) or allergens and the replacement of animal testing. In most cases, the value of this work is derived from the ability to generate enhanced measurements from extremely small samples (few ml) at low cost.

The use of microfluidics in molecular biology has already been shown to offer benefits such as improved sensitivity and speed of analysis. An example here is a charge based capacitive biosensor for bacteria growth monitoring (BGM) presented in [1]. The sensor chip is implemented in a CMOS process in order to show the applicability of the proposed on-chip capacitive technique for BGM purposes. Another example is an integrated microfluidic-fibre optic device for the detection of agglutination for blood type cross-matching proposed and described by [2]. The device consists of a straight microfluidic channel through which a reacted red blood cell suspension flows through a syringe pump based actuation system.

In general, although there are a number of extremely interesting technologies capable of integrating microfluidics with electronic control and processing [3], few commercial implementations exist. Accelerating the uptake of these technologies is hence a core mission of numerous private and public funding bodies. Solutions for defining efficient non-destructive production test strategies, fault tolerant design features and integrated health monitoring are expected to be important contributions towards this goal [4].

In the field of microsystems, embedded test is now acknowledged to be a crucial requirement within the design space of MEMS-based solutions [5]. Within this work, testability has been shown to be especially important within the design flow for microfluidic systems and their biological applications due to the immaturity of the field and limited understanding of the sources of failure. There has also been work on the testing process for the interconnect infrastructure of microfluidic chips [6]. Here mechanical and fluidic testing apparatus and protocols for characterizing both chip-to-chip and world-to-chip interconnects have been proposed.

The idea of Oscillation-Based-Test (OBT) for analogue integrated circuits was initially presented in [7] where the circuit under test is converted to a circuit that oscillates at a frequency that is a function of the material properties and structural integrity of the system. Faults in the circuit under test can be detected which normally cause a deviation of the oscillation frequency from its nominal value. The validity of this technique was verified through examples such as operational amplifiers and analogue-to-digital converters. OBT has also been evaluated within MEMS-based systems through work on a monolithic magnetometer [8]. The natural frequency of the cantilever has been chosen to be the main input parameter to the test strategy. The tolerance band and detection range in the case of small deviations applied to technological process parameters have been assessed. The potential for using OBT in a DNA sensor array has been discussed in [9] and [10] as well where the modes of sensing and test can both be realised in an oscillator-based structure. The idea of monitoring power supply current $\left(\mathrm{I}_{\mathrm{DDQ}}\right)$ has been applied to an electro-fluidic interface in a FlowFET system [11] where the system under test is fundamentally electronic but applied within a microfluidic environment. Bridging faults within this FlowFET system have been shown to be efficiently detected using this method. A functional test for a continuous FlowFET has also been demonstrated in [11]. The underlying principle is that typical faults, such as 
degraded gate voltages, jammed particles in the channel or leakage will influence the fluid velocity and the duration of fluid charging.

For digital droplet-based microfluidic systems, techniques for defect classification, test planning, and test resource optimization have been presented in [12], whilst in [13] both catastrophic and parametric faults have been detected by electro-statically controlling and tracking the motion of test droplets. The detection of these test droplets is through a simple RC oscillator formed by a pair of driving electrodes with the fluid between them acting as the dielectric. Furthermore, based on the test results, the 2-D EWOD (Electro-Wetting On Dielectric) arrays with open structures can be reconfigured in order to reallocate resources and avoid using the sites marked faulty. A concurrent testing method is also presented in [14], which allows fault detection and biomedical assays to run simultaneously on a microfluidic system.

Another method based on Euler paths is proposed in [15], which maps a digital microfluidic biochip to an undirected graph where a test droplet is routed along the Euler path derived from the graph to pass through all the cells in the array. In [16] a droplet routing technique on a digital microfluidic device is proposed to ensure fault-tolerant behavior. Three typical faults are built into fault models for this device. This method gave efficient solutions to problems like optimal routing, run-time fault diagnosis, and subsequent fault recovery.

The dependability has also improved by using digital microfluidic logic gates. In $[17,18]$ fault diagnosis methods to locate a single defective cell and multiple rows/columns with defective cells in a digital microfluidic array have been presented. These methods utilize digital microfluidic (exclusive-OR/AND) gates to implement an output comparator. An efficient testing and diagnosis methodology for digital microfluidic biochips has also been proposed in [19]. This method is based on a partitioning technique that increases parallelism in testing and diagnosis by exploiting the available sources and sinks which supports the ability to detect and locate single as well as multiple faults in a microfluidic array. An excellent survey of research on design automation and test techniques for digital microfluidic biochips can be found in [20].

The work carried out here is motivated by characterisation studies that have revealed that sensor electrode degradation tends to be a key reliability issue in cell-based biosensors and that correction is not possible through manual cleaning $[3,4]$. Degraded electrodes tend to exhibit much lower signal-to-noise ratio (SNR) or higher interface impedance that in both cases degrades the ability of the system to monitor cellular signals and bio-chemical processes. Both the quality of the sensing interface and the integrity of the bio-chemistry are crucial to the performance and reliability of the system. Monitoring key parameters or reliability indicators for these component parts in addition to the transportation and processing functions is hence an essential feature of future systems. This work complements the international effort to advance the field of embedded test for microfluidic systems and builds on initial work on both an MEA (Micro-Electrode Array) structure and full bio-diagnostic system presented in [21,22].

The rest of the paper is organized as follows: Section 2 briefly describes and references the case study and an electrical model at the circuit-level for the electrolyteelectrode interface which has been developed and characterised based on the theoretical analysis of microelectrodes in [23]. In section 3, theoretical consideration of a mid-frequency oscillation test solution to monitor the surface quality of micro-electrodes is presented, which depends on the sensitivity of the bio-fluidic interface capacitance to degradation, contamination and fouling. To verify this technique, experiments on an MEA were carried out, the results of which are documented in section 4 . Finally, conclusions are drawn in section 5.

\section{Self Test Solution and Fault Modeling}

Design-for-test, fault detection and fault diagnosis have become essential for many technologies to increase dependability and enhance safety and reliability. For microfluidic systems, faults and measurement errors are often indistinguishable and hence the need to move from test strategies that generate deterministic pass/fail data to on-line condition monitoring techniques that generate indicators of evolving faults or conditions that may invalidate a measurement.

In using electrodes to sense either spontaneously generated extracellular potentials or to measure the impedance of target cells, the reliability indicator will be related to the fundamental signal coupling between the ion flow in the electrolyte and the electrode interface. Therefore, the electrical properties of the electrode-electrolyte interface are crucial parameters within fault-free and faulty systems. Analysis has however indicated that degradation of the sensing interface and the health status of bio-chemical species are not trivial parameters to monitor.

To help understand the dependence of system performance on such physical defects, an electrical equivalent model may be used. An electrical model at the circuit-level for the interface has been developed and characterised in [21,22] which is based on the theoretical analysis of microelectrodes in [23]. This model is shown in Figure 1, where $\mathrm{C}_{\mathrm{EDL}}$ is the capacitance of the Electrical Double Layer, $\mathrm{R}_{\mathrm{CT}}$ the charge transfer resistance, $Z_{\mathrm{W}}$ the Warburg impedance due to ion diffusion, $Z_{P}$ the impedance of the porous surface, $R_{S}$ the electrolyte spreading resistance and $C_{X}$ is the parasitic capacitance. The model that has been developed for the electrode-electrolyte interface has been characterised on an MEA platform as illustrated in Figure 2 [24]. 


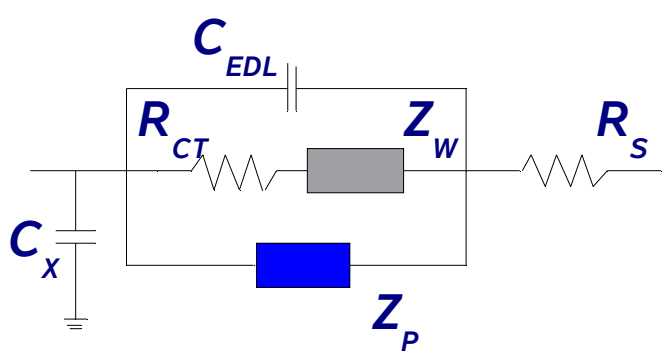

Figure 1 Circuit model for the electrode-electrolyte interface

This is a well-accepted interface for recording the extracellular potentials from neuron networks or cardiac tissues [25]. The MEA has 60 sensing electrodes distributed on a square area of $0.6 \times 0.6 \mathrm{~mm}^{2}$ with the distance between neighbouring electrodes being $200 \mu \mathrm{m}$. Each sensing electrode has a diameter of $30 \mu \mathrm{m}$ and is fabricated using high surface porosity material (TiN). The electrolyte used has a molar concentration of $120 \mathrm{mM}$.

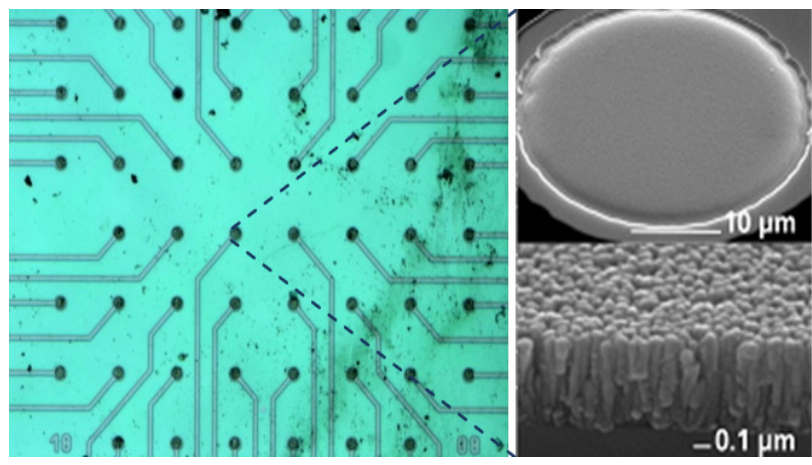

Figure 2 Sensing electrodes site of the MEA (Reproduced from [24])

The values of the components in the interface model have been calculated (Table 1) with good agreement between simulations and experimental results.

Table 1: Calculated values of components in the interface model

\begin{tabular}{l|l}
\hline Component & Value \\
\hline $\mathrm{C}_{\mathrm{EDL}}$ & $125 \mathrm{pF}$ \\
$\mathrm{R}_{\mathrm{CT}}$ & $3 \mathrm{M} \Omega$ \\
$\mathrm{Z}_{\mathrm{W}}$ & $(1-\mathrm{j}) 0.39 / \sqrt{f}$ \\
$\mathrm{Z}_{\mathrm{P}}$ & $(1-\mathrm{j}) 5 \mathrm{e} 6 / \sqrt{f}$ \\
$\mathrm{R}_{\mathrm{S}}$ & $7 \mathrm{~K} \Omega$ \\
$\mathrm{C}_{\mathrm{X}}$ & $2.5 \mathrm{pF}$ \\
\hline
\end{tabular}

For electrodes used within MEAs for recording extracellular signals from neuron networks or cardiac tissues, there are three potential mechanisms that can result in quality degradation of micro-electrodes according to the end-users. They are:

- electrode degradation due to damage during the cleaning and preparation of the MEAs,

- fouling of the electrodes by biological material that is not removed completely during cleaning, and

- material loss of the electrode surface through ion loss into the surrounding liquid medium.

All of these affect the electrode-electrolyte interface in the form of either physical damage, fouling from biological material deposits, or loss of solid material from the electrode surface. As the porous layer is located on top of the micro-electrodes and interfaced with the operational environment, it is logical to assume that the fault mechanism active will degrade the performance of the system through damage to the porous layer to some degree.

\section{Oscillation-Based Technique (OBT)}

\subsection{Surface Degradation and the Electrical Double Layer (EDL)}

The model presented in Figure 1 has been shown to be useful for exploring the effect of surface degradation of the bio-electrodes under study. In many implementations of electrodes and electrode arrays, the value of $\mathrm{C}_{\mathrm{EDL}}$ is a function of both expected biological processes (e.g. hybridisation) and degradation mechanisms such as fouling, contamination or electrolysis due to voltage bias. The electrode surface was made from high porosity material to increase the effect of the EDL. It is hence logical to expect that all relevant types of degradation that will affect the electrode structure will directly impact this layer. Figure 3 shows two different examples of degradation on the electrode surface area.

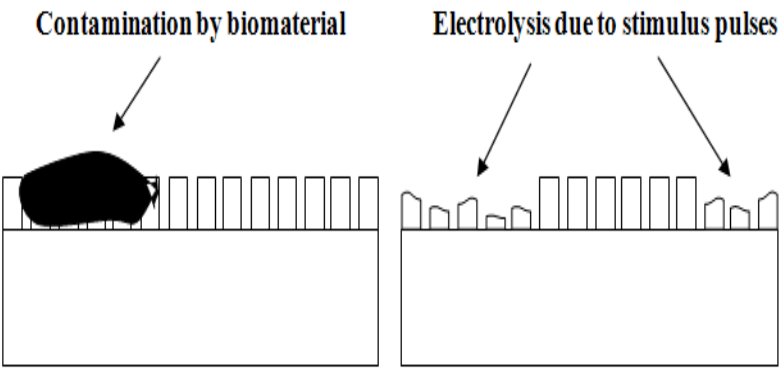

Figure 3 Examples of degradation mechanisms on the electrode surface

When a solid (metal, semiconductor, insulator) is placed into an electrolyte (ionic solution), an electrified interface is formed due to chemical reactions where electrons are transferred between the solid and the electrolyte. The result is the formation of an electric field between the electrode and the electrolyte which stimulates further chemical reactions to take place. These reactions reach an 
equilibrium condition when the currents due to electron transfer to and from the solid are equal. The result of this process is the Electrical Double Layer (EDL).

This EDL therefore originates from spontaneously formed surface charge near the electrode/electrolyte interface which supports a net excess of mobile ions with a polarity opposite to that of the surface. Equilibrium is reached when sufficient charge separation is achieved at the interface. Consequently, two layers are formed at the electrode surface, namely the compact layer and the diffusion layer. Two key mechanisms manifest themselves in the electrode/electrolyte interface; capacitive effects, which dominate when voltages across the interface are small (as for neural signal recording), and a resistive effect which dominates when significant current flows. Electrodes are typically connected to the inputs of extremely high impedance amplifier circuits when used to record neural signals. This mode of operation involves mainly the capacitive properties of the electrodes. When used for the stimulation of neural signals where sufficient current flow is required, electrodes behave resistively. In this case electrochemical mechanisms are important.

Looking in more detail at the capacitive component of the EDL, it can be shown that $\mathrm{C}_{\mathrm{EDL}}$ can be modelled by an equivalent network consisting of $\mathrm{C}_{\mathrm{H}}$, the compact layer capacitance, $C_{D}$, the diffusion capacitance, $R_{D}$, the diffusion layer resistance and $\mathrm{R}_{\mathrm{B}}$, the bulk resistance, as shown in Figure 4. The resistance effect in the compact layer has been neglected because the ions in this layer are to a first approximation immobilized. Table 2 shows the calculated values for the EDL components.

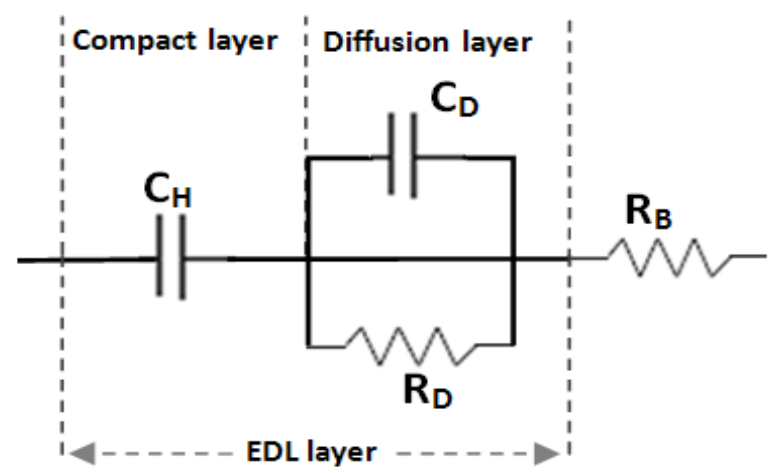

Figure 4 Extension of the electrode model to include components of $C_{E D L}$ which can be replaced by $C_{H}, C_{D}, R_{D}$ and $\mathbf{R}_{B}$

Table 2: Calculated values of EDL components

\begin{tabular}{l|l}
\hline Component & Value \\
\hline $\mathrm{C}_{\mathrm{H}}$ & $125 \mathrm{pF}$ \\
$\mathrm{C}_{\mathrm{D}}$ & $\geq 758 \mathrm{pF}$ \\
$\mathrm{R}_{\mathrm{D}}$ & $3 \mathrm{M} \Omega$ \\
$\mathrm{R}_{\mathrm{B}}$ & $1 \mathrm{~K} \Omega$ \\
\hline
\end{tabular}

The interface dominant capacitance of the EDL is $\mathrm{C}_{\mathrm{H}}$ and can be approximately expressed as

$$
C_{H}=\varepsilon_{o} \varepsilon_{r} A / X_{1}
$$

where $\varepsilon_{o}$ is the permittivity of free space, $\varepsilon_{r}$ is the relative permittivity of the electrolyte and $X_{1}$ is the length of the compact capacitor (dominant capacitor), while $\mathrm{A}$ is the surface area of the electrode. As discussed above, damage to the porous layer on the surface of the sensing electrodes is an important physical defect which results from a range of electrochemical, bio-chemical and electromagnetic effects. It is also noted that the surface area of the electrode can be used to assess degradation because all key mechanisms will affect the active area and consequently change the dominant capacitance, $\mathrm{C}_{\mathrm{H}}$. Based on the model above which describes the EDL at the electrode/electrolyte interface, the health status of the porous layer can be monitored as a function of frequency. The values of the parameters for the equivalent circuit are shown in Table 2 and to consider the effect of the porous material on the EDL, it is assumed that the porous layer will produce a 10 times larger effective surface area than a similar flat electrode.

\subsection{Simulation Results}

To study the impact of the effective surface area of the electrode on the oscillation frequency of a test circuit, $\mathrm{C}_{\mathrm{EDL}}$ was inserted into the feedback of a standard oscillator structure shown in Figure 5.

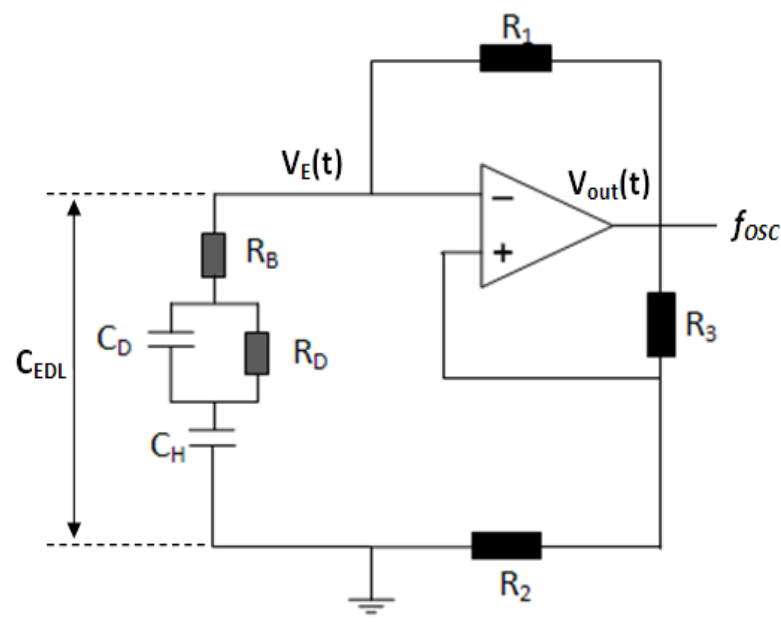

Figure $5 \mathrm{C}_{\mathrm{EDL}}$ Integrated into the oscillation structure

The value of the compact layer capacitance has been varied to correspond to changes in an effective surface area between $1 \%$ and $100 \%$ of the nominal value. Figure 6 shows the simulation results where it is observed that the decrease in effective surface area of the electrode will significantly impact the oscillation frequency. The oscillator circuit used for characterisation of the effectiveness of OBT has used values for $R_{1}, R_{2}$, and $R_{3}$ of $1 \mathrm{M}, 1 \mathrm{~K}$ and $100 \mathrm{~K} \Omega$ respectively. 


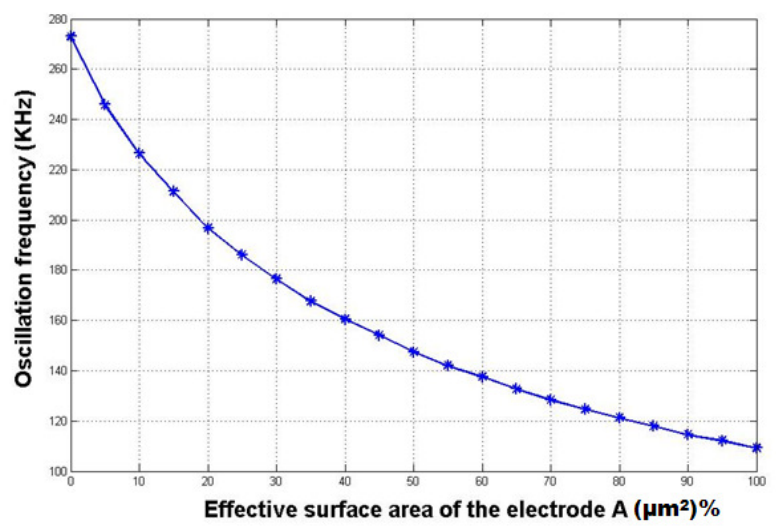

Figure 6 Oscillation frequencies as a function of electrode surface area

The reasons for choosing these values are to maximise the sensitivity to changes in $\mathrm{C}_{\mathrm{H}}$ and to avoid electrode damage from the feedback voltage. The feedback voltage $\left(\mathrm{V}_{\mathrm{E}}\right)$ can be expressed as follows:

$$
\begin{aligned}
& V_{E}(t)=V_{\text {out }}(t) \times \frac{R_{2}}{R_{2}+R_{3}}+k_{E}(t) \\
& V_{\text {out }}(t)=V_{O}+k_{\text {out }}(t)
\end{aligned}
$$

where $\mathrm{V}_{\mathrm{O}}$ is the power supply voltage of the op-amp, $\mathrm{k}_{\mathrm{E}}(\mathrm{t})$ and $\mathrm{k}_{\mathrm{out}}(\mathrm{t})$ are the slopes of the linearly increased or decreased voltages $V_{E}(t)$ and $V_{\text {out }}(t)$ respectively. Figure 7 shows the ideal output waveform of the oscillator and the related voltages.

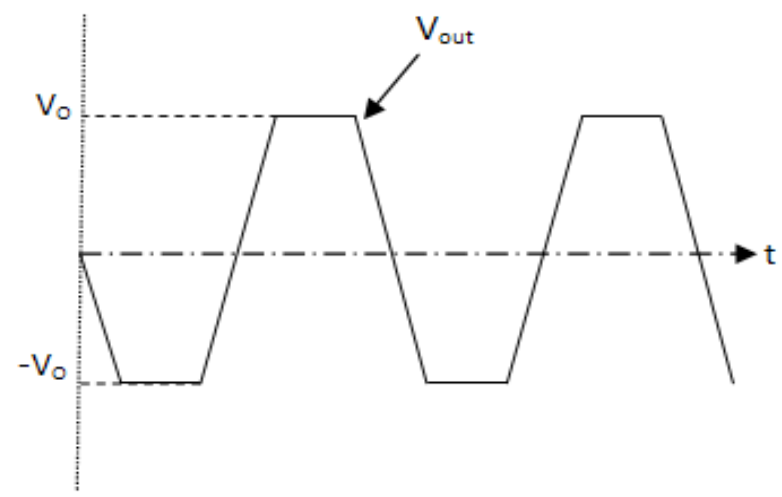

Figure 7 The ideal output waveform of the oscillator

With this set of values, the feedback voltage on the electrode is around $\pm 100 \mathrm{mV}$, and will be the same amplitude and the same duration but opposite polarity. Therefore, the accumulated charge will flow back to the op-amp circuit and not held at the electrode. The nominal value of $C_{H}$ is assumed to be $125 \mathrm{pF}$, while $C_{D}, R_{D}$ and $R_{B}$ are assumed to be constant because the change in electrode surface area has no effect. This oscillator outputs a square wave at a frequency of around $110 \mathrm{KHz}$ in the fault-free case, and between $110 \mathrm{KHz}$ and $250 \mathrm{KHz}$ dependant on the change in active area.

To check the effect of the other parameters in the $\mathrm{C}_{\mathrm{EDL}}$ branch on oscillation frequency and verify that $\mathrm{C}_{\mathrm{H}}$ is the dominant parameter, an initial exploration of the sensitivity of the method to changes in $\mathrm{C}_{\mathrm{EDL}}$ components has been carried out. All values have been swept from nominal to $50 \%$ of their values as shown in Table 3 , where the oscillation frequency has increased from $109 \mathrm{KHz}$ to $147 \mathrm{KHz}$ as a function of $\mathrm{C}_{\mathrm{H}}$ whilst the increase is only to around $117 \mathrm{KHz}$ under changes to other parameters with $\mathrm{C}_{\mathrm{H}}$ constant.

Table 3: Effect of change all $\mathrm{C}_{\mathrm{EDL}}$ parameters on the oscillation frequency

\begin{tabular}{cccccc}
\hline $\begin{array}{c}\text { Test } \\
\text { case }\end{array}$ & $\begin{array}{c}\mathrm{C}_{\mathrm{H}} \\
(\mathrm{pf})\end{array}$ & $\begin{array}{c}\mathrm{C}_{\mathrm{D}} \\
(\mathrm{pf})\end{array}$ & $\begin{array}{c}\mathrm{R}_{\mathrm{D}} \\
(\mathrm{M} \Omega)\end{array}$ & $\begin{array}{c}\mathrm{R}_{\mathrm{B}} \\
(\mathrm{K} \Omega)\end{array}$ & $\begin{array}{c}\text { Oscillation } \\
\text { frequency } \\
(\mathrm{KHz})\end{array}$ \\
\hline 1 & $125^{*}$ & $800^{*}$ & $3^{*}$ & $1^{*}$ & 109.28 \\
2 & 93.75 & 800 & 3 & 1 & 124.59 \\
3 & 62.50 & 800 & 3 & 1 & 147.34 \\
4 & 125 & 600 & 3 & 1 & 111.65 \\
5 & 125 & 400 & 3 & 1 & 117.31 \\
6 & 125 & 800 & 2.25 & 1 & 109.22 \\
7 & 125 & 800 & 1.5 & 1 & 109.86 \\
8 & 125 & 800 & 3 & 0.75 & 108.08 \\
9 & 125 & 800 & 3 & 0.50 & 107.58 \\
10 & 125 & 600 & 2.25 & 0.75 & 111.32 \\
11 & 125 & 400 & 1.50 & 0.50 & 115.36 \\
\hline
\end{tabular}

* refers to $\mathrm{C}_{\mathrm{EDL}}$ nominal values

\section{Experimental Results}

\subsection{EDL Capacitance and Measurement Deviation}

To verify the oscillation-based self-test solution, an experiment on an MEA demonstrator has been carried out. This MEA has 60 planer electrodes fabricated on a glass substrate. The electrode surface is sputtered with TiN forming a mechanically stable columnar structure in order to increase the effective surface area. In this experiment, the EDL capacitance is measured directly using a Wayne Kerr Precision Impedance Analyzer 6500. Measurements are conducted by connecting the impedance analyser to the target electrode and the ground electrode. In the set-up, the ground electrode serving as an internal reference is shorted with the electrolyte bath ground, and connected to the electronics ground in order to provide a common ground between the electronics and the bath.

To check and demonstrate the deviation in the EDL capacitance for the fault-free electrodes, $\mathrm{C}_{\mathrm{EDL}}$ readings for ten fault-free electrodes were captured as shown in Table 4. The nominal value for $\mathrm{C}_{\mathrm{EDL}}$ has been assumed to 
be $180.64 \mathrm{pF}$, and as identified in the measurements, the maximum deviation is less than $\pm 5 \%$. The capacitance is measured for the condition where the system is stimulated with a $50 \mathrm{mV}$ input at $10 \mathrm{KHz}$.

Table 4: $C_{E D L}$ readings for randomly selected fault-free electrodes

\begin{tabular}{ccc}
\hline Electrode No. & $\mathrm{C}_{\mathrm{EDL}}(\mathrm{pf})$ & Deviation \\
\hline 75 & 180.640 & Nominal \\
85 & 180.144 & $-0.2747 \%$ \\
27 & 178.560 & $-1.1518 \%$ \\
46 & 176.704 & $-2.1790 \%$ \\
26 & 189.394 & $+4.8459 \%$ \\
38 & 178.779 & $-1.0305 \%$ \\
53 & 180.173 & $-0.2587 \%$ \\
74 & 187.815 & $+3.9720 \%$ \\
17 & 189.323 & $+4.8067 \%$ \\
51 & 187.160 & $+3.6091 \%$ \\
\hline
\end{tabular}

\subsection{Degradation Effect on EDL Capacitance and Oscillation Frequency}

To demonstrate the effect of the electrode surface degradation on the EDL capacitance and to extract $\mathrm{C}_{\mathrm{EDL}}$ measurements at different levels of degradation, another experiment on an MEA has been carried out. The electrode surface has been artificially damaged by repeatedly stimulating the electrodes with voltage pulses (1 Volt). Under these conditions the stimulating electrode behaves as a plate capacitor with the accumulated charge maintained on the surface resulting in time dependant degradation due to electrolysis [24]. Figures 8 and 9 show three-dimensional images for fault-free and artificially damaged electrodes respectively. These images have been captured using a Veeco Wyko NT100 White Light Interferometer. As can be seen from these figures, the effect of the stress pulses on the electrode surface area is the loss of large areas of the porous layer due to electrolysis.

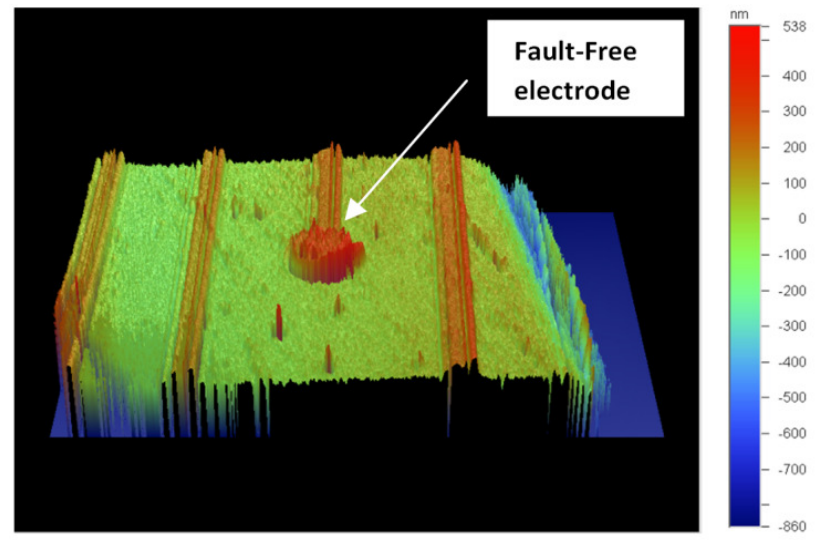

Figure 8 3-D interactive image for fault-free electrode

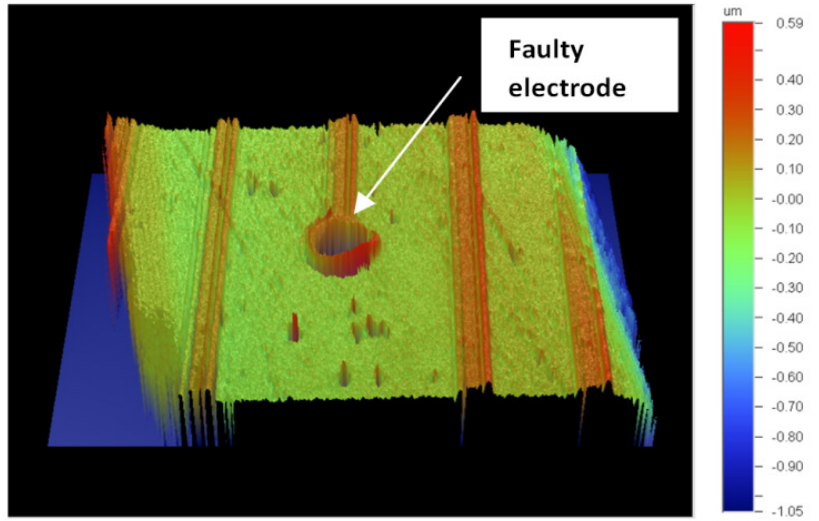

Figure 9 3-D interactive image for faulty electrode after application of stress pulses

This damage impacts the functionality of the surface porous layer and is the most widely reported degradation mechanism in typical applications. The EDL capacitance has been measured after specific time points within the stress program to extract the $\mathrm{C}_{\mathrm{EDL}}$ signature as a function of pulse time. The period of the stress pulse is 5 seconds with a stress program duration of 150 seconds. By stimulating the electrode with these pulses $\mathrm{C}_{\mathrm{EDL}}$ has decreased from around $180 \mathrm{pF}$ to $55 \mathrm{pF}$. As a consequence, the oscillation frequency has increased from $78 \mathrm{KHz}$ to about $150 \mathrm{KHz}$. Table 5 shows the change in the stress pulse time, $\mathrm{C}_{\mathrm{EDL}}$ and the oscillation frequency over the duration of the stress program.

Table 5: The change in $C_{E D L}$ and the oscillation frequency with the stress pulse time

\begin{tabular}{|c|c|c|}
\hline $\begin{array}{l}\text { Stress pulse time } \\
\text { (s) }\end{array}$ & $\mathrm{C}_{\mathrm{EDL}}(\mathrm{pF})$ & $\begin{array}{c}\text { Oscillation } \\
\text { frequency } \\
(\mathrm{KHz})\end{array}$ \\
\hline 0 & 180.6 & 78.19 \\
\hline 5 & 143.7 & 89.92 \\
\hline 10 & 136.1 & 92.79 \\
\hline 15 & 121.5 & 99.32 \\
\hline 20 & 101.3 & 110.12 \\
\hline 25 & 97.8 & 112.93 \\
\hline 30 & 95.2 & 114.12 \\
\hline 35 & 91.7 & 116.61 \\
\hline 40 & 88.4 & 118.73 \\
\hline 45 & 87.6 & 119.13 \\
\hline 50 & 81.9 & 122.26 \\
\hline 60 & 76.2 & 127.86 \\
\hline 70 & 72.4 & 131.33 \\
\hline 80 & 68.7 & 134.57 \\
\hline 90 & 63.6 & 139.18 \\
\hline 100 & 59.3 & 144.27 \\
\hline 120 & 57.2 & 145.51 \\
\hline 150 & 55.1 & 148.36 \\
\hline
\end{tabular}


Comparing the oscillation frequency measurements in Table 5 with the expected simulation results (Figure 6), a difference in the frequency range can be observed. This can be related to the following: Firstly, the electrode surface area is made from high porosity material (TiN). It is difficult to calculate this area exactly due to its microstructure. Secondly, there is a difference between the input impedance of the op-amp in the simulation and the experimental test bench. In addition, there is the effect of the parasitic capacitance, electrolyte spreading resistance and the difference between the physical behaviour in the experiments and simulation work. As a consequence, all these factors will affect the frequency range, but the main goal is still valid because the changes in the measurement between the fault-free and faulty cases are doubled for the whole frequency range.

\subsection{OBT Implementation at the System Level}

To monitor the condition of the micro electrode array and verify the effectiveness of OBT in the system-level design, this technique has been applied to a device with 32 microelectrodes. The system consists of a control unit, an analogue multiplexer, and the test circuit. The whole system architecture is shown in figure 10 .

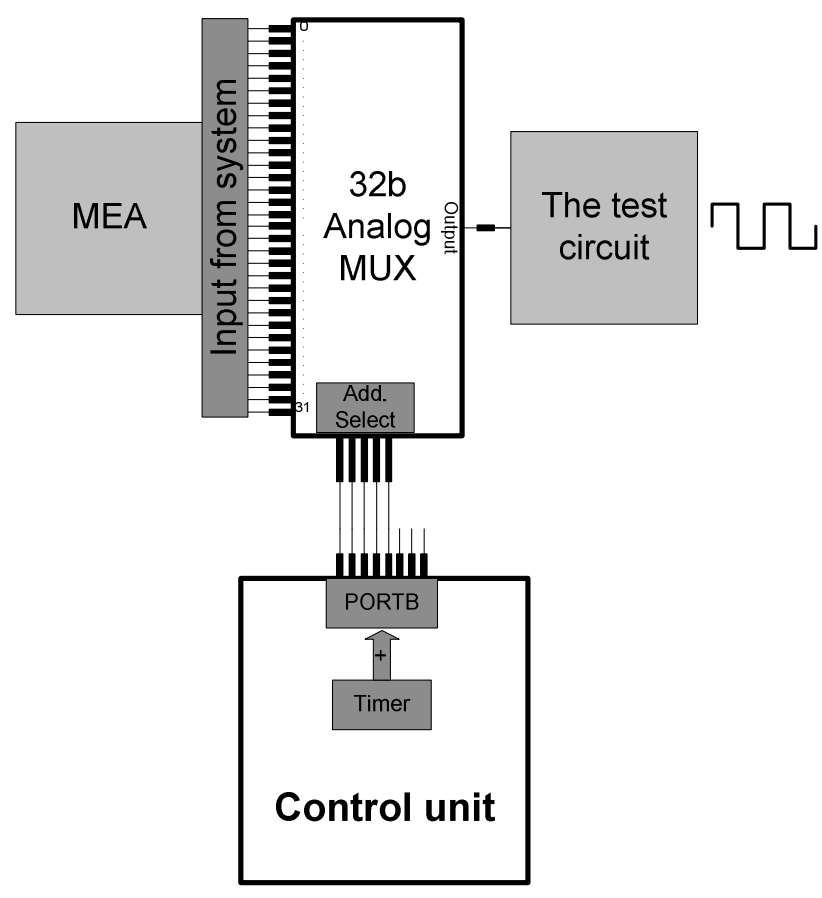

Figure 10 The system architecture

The oscillation circuit in figure 5 is used to characterize the effectiveness of the OBT and study the effect of the system-level parameters on the output frequency in the fault-free and faulty cases. An ADG732 analogue multiplexer (monolithic CMOS 32-channel) has been used to connect the micro electrode array (32 electrodes from the MEA) to the oscillator test circuit. This multiplexer switches the inputs (S0-S31) to a common output as determined by the 5-bit binary address lines (A0-A4). The control unit is responsible for synchronization of the whole system. An Atmel megaAVR series microcontroller has been used for this purpose. The ATmega8A is a low-power CMOS 8-bit microcontroller based on the AVR RISC architecture which is programmed in " $C$ " to increment the output port after a defined delay using the built-in timer. The output port is limited to the 16-bit address range of the AMUX IC so only valid addresses can be selected on the AMUX. This particular microcontroller could uniquely address up to eight 16-bit AMUX ICs simultaneously.

The system provides on-line monitoring of the degradation states of 32 electrodes, and it is triggered by a control signal from the microcontroller. Depending on the control signal, the multiplexer connects each electrode for a certain time with the test circuit. This utilizes the output port, and the address lines. The test circuit converts the EDL capacitance to a frequency, which when compared with a nominal value, indicates the degradation level of the electrode under test. Figure 11 shows the code flow for the test algorithm.

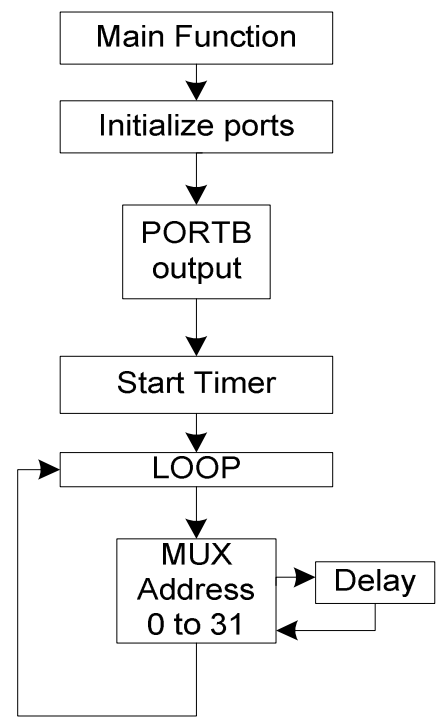

Figure 11 The code-flow for the test algorithm

To validate the test technique and illustrate the effect of the system parameters, an experiment on the MEA has been carried out. Thirty-two electrodes from the MEA were connected through the multiplexer to the test circuit. Different levels of degradation have been emulated by artificially damaging the surface area of ten electrodes with voltage stress pulses of $1 \mathrm{~V}$ amplitude and differing periods. The microcontroller has been programmed to increment output port B and a delay time of two seconds has been used to connect each electrode to the oscillator. Figure 12 shows the frequency measurements where the test technique has accurately distinguished the degraded electrodes and the level of the degradation. The fault-free frequency is denoted by $f_{\text {osc }}$. 


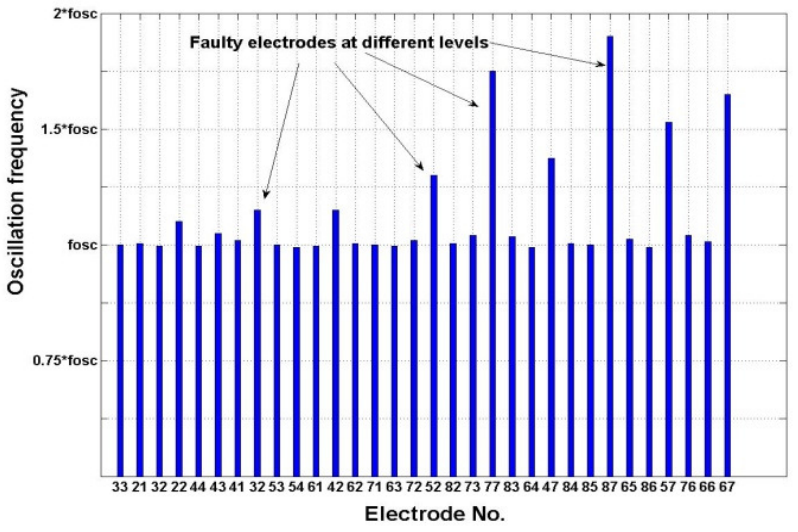

Figure 12 Oscillation frequency measurements for 32 electrodes

To demonstrate the effect of the analogue multiplexer input capacitance and resistance on the measurements, the oscillation frequency of ten electrodes (the same electrodes degraded by varying stress inputs) have been measured with and without the multiplexer. Single wires have been used in this experiment to avoid the coupling capacitance of the ribbon cable (Table 6). The effect of the self resistance, self capacitance and the coupling capacitance on the frequency measurements has also been demonstrated in a second experiment where the single wires have been replaced with ribbon cable. The equivalent circuit per unit length for the interface between the electrodes and the analogue multiplexer can be seen in Figure 13 , where $\mathrm{R}_{\mathrm{S}}, \mathrm{C}_{\mathrm{S}}, \mathrm{C}_{\mathrm{C}}$, and $\mathrm{N}$ are the self resistance, the self capacitance, the coupling capacitance, and the line numbers of the ribbon cable.

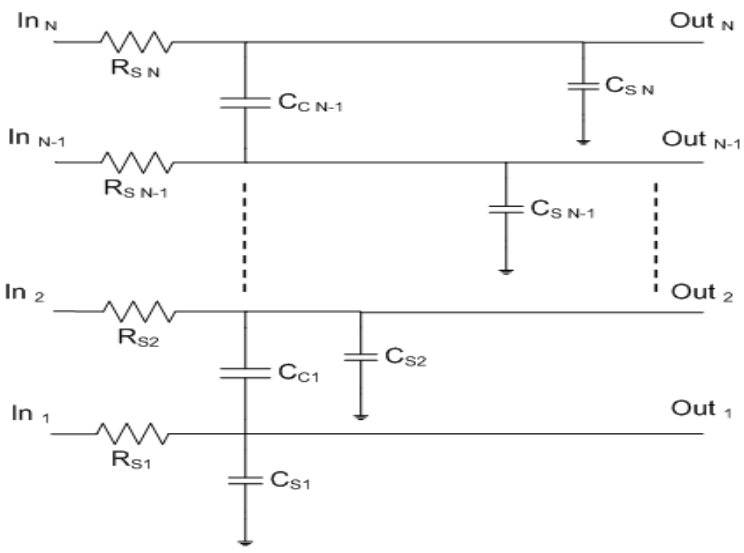

Figure 13 The per unit length electrical equivalent circuit for the ribbon cable

Table 6 shows the oscillation frequency measurements when single wires without the AMUX were used, and then when AMUX was inserted. The last column shows the measurements when the single wires have been replaced by ribbon cable.
Table 6: Oscillation frequency measurements using single wires and ribbon cable with and without AMUX

\begin{tabular}{ccccc}
\hline $\begin{array}{c}\text { Electrode } \\
\text { No }\end{array}$ & $\begin{array}{c}\text { Stress } \\
\text { pulse } \\
\text { time } \\
(\mathrm{s})\end{array}$ & $\begin{array}{c}\text { Oscillation } \\
\text { frequency } \\
\text { without } \\
\text { AMUX } \\
\text { using } \\
\text { single } \\
\text { wires }\end{array}$ & $\begin{array}{c}\text { Oscillation } \\
\text { frequency } \\
\text { with AMUX } \\
\text { using single } \\
\text { wires }\end{array}$ & $\begin{array}{c}\text { Oscillation } \\
\text { frequency with } \\
\text { AMUX using } \\
\text { ribbon cable }\end{array}$ \\
\hline 21 & 0 & $f_{\text {osc }}$ & $0.93 f_{\text {osc }}=f_{\text {osc }}{ }^{*}$ & $0.88 f_{\text {osc }}=f_{\text {osc }}{ }^{* *}$ \\
22 & 5 & $1.15 f_{\text {osc }}$ & $1.12 f_{\text {osc }}{ }^{*}$ & $1.12 f_{\text {osc }}{ }^{* *}$ \\
32 & 10 & $1.20 f_{\text {osc }}$ & $1.18 f_{\text {osc }}{ }^{*}$ & $1.17 f_{\text {osc }}{ }^{* *}$ \\
42 & 15 & $1.27 f_{\text {osc }}$ & $1.25 f_{\text {osc }}{ }^{*}$ & $1.22 f_{\text {osc }}{ }^{* *}$ \\
52 & 20 & $1.41 f_{\text {osc }}$ & $1.40 f_{\text {osc }}{ }^{*}$ & $1.36 f_{\text {osc }}{ }^{* *}$ \\
47 & 30 & $1.47 f_{\text {osc }}$ & $1.45 f_{\text {osc }}{ }^{*}$ & $1.44 f_{\text {osc }}{ }^{* *}$ \\
57 & 50 & $1.56 f_{\text {osc }}$ & $1.53 f_{\text {osc }}{ }^{*}$ & $1.50 f_{\text {osc }}{ }^{* *}$ \\
67 & 70 & $1.68 f_{\text {osc }}$ & $1.65 f_{\text {osc }}{ }^{*}$ & $1.59 f_{\text {osc }}{ }^{* *}$ \\
77 & 100 & $1.85 f_{\text {osc }}$ & $1.76 f_{\text {osc }}{ }^{*}$ & $1.68 f_{\text {osc }}{ }^{* *}$ \\
87 & 150 & $1.93 f_{\text {osc }}$ & $1.82 f_{\text {osc }}{ }^{*}$ & $1.75 f_{\text {osc }}{ }^{* *}$ \\
& & &
\end{tabular}

The effect of the multiplexer input parameters (on-state capacitance and resistance) can be observed from the table where the normal oscillation frequency and modified frequencies due to different degradation levels have been shifted from their nominal values. The fault-free frequency has decreased by $7 \%$ while the full-range of the output frequency between the fault-free and the faulty cases has also decreased from $1.93 \%$ without AMUX to around $1.82 \%$ with the AMUX.

The ribbon cable parameters have also affected the measurements, where the fault-free oscillation frequency has decreased by $12 \%$. The full-range measurements have also been limited by the self and coupling capacitances from $1.82 \%$ with single wires to around $1.75 \%$ with ribbon cable.

To check and validate the test technique for other types of defects, three electrodes were chosen to verify the effectiveness for short and open circuits. Electrodes 57 and 58 have been shorted at the contact pins while an open circuit has been made by splitting the contact pin from the contact pad for electrode number 63 . In the first case, the oscillation frequency has decreased to around $30 \%$ of its normal value because the effective surface area has physically doubled due to the short between the electrodes. In the second case, and due to the open circuit between the contact pin and the contact pad, the output frequency has increased to around $230 \%$ of its normal value. This suggests that this test technique can easily detect the faulty electrodes at different levels of degradation, and distinguish them from other different types of faults due to the frequency measurements and their deviation from the normal value. 


\section{Conclusions}

An oscillation-based test and diagnosis technique for an electrode structure has been presented which is based on the sensitivity of the bio-fluidic interface capacitance to degradation, contamination and fouling. The technique has the potential to be built into integrated bio-fluidic systems. The design of the monitoring structure has been evaluated through physical characterisation involving data extracted for both a single electrode and an array of electrodes. The technique provides an analogue value directly related to the degradation of the electrode, so has the ability to provide on-line degradation detection for a range of conditions on the electrode surface.

The validation of the on-line application of this solution to a normal operating MEA, and the effect of the feedback voltage on the measurements, requires further study. In addition, the possibility of using this technique to detect other types of degradation needs to be considered.

Future work will involve the further development of architectures where the integration of this technique into a higher level control system will support adaptability to ensure defective electrodes can be switched out of the system and the array reconfigured to maintain functionality. Of further importance is how electrode monitoring strategies can be integrated with other sensors and test solutions within a portable instrument.

\section{Acknowledgments}

The Authors would like to thank the EPSRC Innovative Electronics Manufacturing Centre "I-Health" project and the FP6 "INTEGRAMplus" program. We would also like to thank Dr Norbert Dumas (University Montpellier) and Prof. Hans Kerkhoff (Uni. Twente) for valuable input, and Dr Richard Heal (QinetiQ) for supplying the MEA platform.

\section{References}

[1] E.G. Zadeh, M. Sawan, A. Shabani, M. Zourob, and V. Chodavarapu, "Bacteria growth monitoring through an on-chip capacitive sensor", Proceedings of $14^{\text {th }}$ IEEE International Mixed-Signal Test Workshop, 2008.

[2] M.K. Ramasubramanian and S.P. Alexander, "An integrated fiberoptic-microfluidic device for agglutination detection and blood typing", Biomed Microdevices, vol. 11, 2009, pp. 217-229.

[3] S. Fei, K. Chakrabarty, and R.B. Fair, "Microfluidicsbased biochips: technology issues, implementation platforms, and design-automation challenges", IEEE Trans on Computer-Aided Design, vol. 25, 2006, pp. 211-223.

[4] H.G. Kerkhoff, "Testing of microelectronic-biofluidic systems", IEEE D\&T for Computer, vol. 24, 2007, pp. 72-82.
[5] H.G. Kerkhoff, "Testing of MEMS-based microsystems", Proceedings of 10th IEEE European Test Symposium, 2005, pp. 223-228.

[6] B.L. Gray, S. Jaffer, D.G. Sahota, and S.M. Westwood, "Mechanical and fluidic characterization of microfluidic interconnects for lab-on-a-chip applications", Proceedings of 14th IEEE International Mixed-Signal Test Workshop, 2008.

[7] K. Arabi and B. Kaminska, "Oscillation-test strategy for analog and mixed-signal integrated circuits", Proceedings of 14th VLSI Symposium, 1996, pp. 476482.

[8] V. Beroulle, Y. Bertrand, L. Latorre, and P. Nouet, "Evaluation of the oscillation-based test methodology for micro-electro-mechanical systems", Proceedings of 20th IEEE VLSI Test Symposium, 2002, pp. 439444.

[9] H. Liu, H.G. Kerkhoff, A. Richardson, X. Zhang, P. Nouet, and F. Azais, "Testing and design of an oscillation-based system architecture for DNA sensor array", Proceedings of 11th International MixedSignal Test Workshop, 2005, pp. 234-239.

[10]H.G. Kerkhoff, X. Zhang, H. Liu, A. Richardson, P. Nouet, and F. Azais, "VHDL-AMS Fault simulation for testing DNA bio-sensing arrays", Proceedings of 4th IEEE Conference on Sensors, 2005, pp. 10301033.

[11]H.G. Kerkhoff and M. Acar, "Testable design and testing of micro-electro-fluidic arrays", Proceedings of the 21st IEEE VLSI Test Symposium, 2003, pp. 403-409.

[12]F. Su, S. Ozev, and K. Chakrabarty, "Testing of droplet-based microelectrofluidic systems", Proceedings of the International Test Conference, 2003, pp. 1192-1200.

[13]F. Su, S. Ozev, and K. Chakrabarty, "Ensuring the operational health of droplet-based microelectrofluidic biosensor systems", IEEE Sensors Journal, vol. 5(4), 2005, pp. 763-773.

[14]F. Su, S. Ozev, and K. Chakrabarty, "Concurrent testing of droplet-based microfluidic systems for multiplexed biomedical assays", Proceedings of the International Test Conference, 2004, pp. 883-892.

[15]F. Su, W. Hwang, A. Mukherjee, and K. Chakrabarty, "Defect-oriented testing and diagnosis of digital microfluidics-based biochips", Proceedings of IEEE International Test Conference, 2005, pp. 487-496.

[16]X. Zhang, F. Proosdij, and H.G. Kerkhoff, "A droplet routing technique for fault-tolerant digital microfluidic devices", Proceedings of 14th IEEE International Mixed-Signal Test Workshop, 2008.

[17] Y. Zhao and K. Chakrabarty, "Fault diagnosis for labon-chip using digital microfluidic logic gates", Proceedings of 14th IEEE International Mixed-Signal Test Workshop, 2008. 
[18] Y. Zhao and K. Chakrabarty, "On-line testing of labon-chip using reconfigurable digital-microfluidic compactors", Int J. Parallel Prog., vol. 37, 2009, pp. 370-388.

[19]S. Datta, B. Joshi, A. Ravindran, and A. Mukherjee, "Efficient parallel testing and diagnosis of digital microfluidic biochips", ACM J. Emerg. Technol. Comput. Syst. Vol. 5, 2, 2009, Article 10.

[20]K. Chakrabarty, "Design automation and test solutions for digital microfluidic biochips", IEEE Transactions on Circuits and Systems, vol. 57, 2010, pp. 4-17.

[21]H. Liu, A. Richardson, T. Harvey, T. Ryan, and C. Pickering, "Embedded test \& health monitoring strategies for bio-fluidic microsystems", Proceedings of the IEEE Electronic Systems Technology Conference, 2008, pp. 463-469.

[22]Q. Al-Gayem, H. Liu, A. Richardson, and N. Burd, "Built-in test solutions for the electrode structures in bio-fluidic microsystems", Proceedings of the IEEE European Test Symposium, 2009, pp. 73-78.

[23]T.A.K. Gregory, Chapter 7 in "Enabling technologies for cultured neural networks", Academic Press, 1994, ISBN 978-0126659702.

[24]Multi-Channel Systems, GmbH, Micro-Electrode Array (MEA) User Manual. 2005, Aspenhaustraße 21, $72770 \quad$ Reutlingen, Germany, http://www.multichannelsystems.com/.

[25]A. Stett, U. Egert, E. Guenther, F. Hofmann, T. Meyer, W. Nisch, and H. Haemmerle, "Biological application of microelectrode arrays in drug discovery and basic research", Analytical and Bioanalytical Chemistry, vol. 377(3), 2003, pp. 486-495. 